\title{
Delivering joined-up care for people with type 2 diabetes: rationale, challenges and examples
}

\author{
STEVE BAIN, ${ }^{1}$ ALICE RICKFORD,${ }^{2}$ NEIL BLACK ${ }^{3}$ LESLEY HAMILTON, ${ }^{4}$ ELIZABETH CAMFIELD, ${ }^{5}$ \\ MARK CHAMLEY, ${ }^{2}$ ANNA HODGKINSON, ${ }^{2}$ JANAKA KARALLIEDDE $^{6}$
}

\begin{abstract}
Approximately $\mathbf{3 . 8}$ million people in the UK have type 2 diabetes mellitus (T2DM) and are, as a consequence, at risk of developing micro- and macrovascular disease. The rapid increase in T2DM prevalence places a considerable burden on secondary healthcare. New classes of glucose-lowering medications (sodium-glucose co-transporter 2 inhibitors and glucagon-like peptide 1 receptor agonists) can improve macrovascular outcomes for people with T2DM; however, these options have brought another layer of complexity to managing this disease. In combination, these factors are calling into question the suitability of the primary-secondary care healthcare model and prompting healthcare professionals to investigate alternative solutions. Bringing high-quality care to people with diabetes and meeting their complex needs requires integrated multidisciplinary expertise in the community. However, setting up such systems within the National Health Service (NHS) can be challenging. The complexities of the internal market (in NHS England), lack of training and expertise, inadequate information technology systems and resistance to change on a systemic and individual level all constitute significant barriers to establishing easy-to-access integrated care. When barriers are removed, successful integrated care systems can be established, which
\end{abstract}

Institute of Life Science, Swansea University Medical School, Singleton Park, Swansea, UK

2 Lambeth Diabetes Integrated Care Team, Crown Dale Medical Centre, London, UK

3 Department of Endocrinology and Diabetes, Altnagelvin Hospital, Derry/Londonderry, UK

4 Western Health and Social Care Trust, Altnagelvin Area Hospital site, Derry/Londonderry, UK

Guy's and St Thomas' NHS Foundation Trust, UK

6 School of Cardiovascular Medicine and Sciences, King's College London, London, UK

Address for correspondence: Dr Steve Bain Institute of Life Science, Swansea University Medical School, Singleton Park, Swansea, SA2 8PP, UK

E-mail: s.c.bain@swansea.ac.uk

https://doi.org/10.15277/bjd.2021.294 improve care for people with diabetes and alleviate pressure on secondary care centres.

Br J Diabetes 2021;21:89-95

Key words: type 2 diabetes, cardiovascular disease, chronic kidney disease, multidisciplinary care, general practice

\section{Background}

Evolution of care for patients with type 2 diabetes

Diabetes is now seen as a modern pandemic. In 2017 there were estimated to be 58 million people in Europe with diabetes, a figure which is set to rise to 67 million within the next 25 years. ${ }^{1}$ Over $90 \%$ of adults with diabetes have type 2 diabetes mellitus $(\mathrm{T} 2 \mathrm{DM}){ }^{2-4}$ the prevalence of which is increasing as a consequence of an ageing population, changes in diet, increasing obesity and more sedentary lifestyles. ${ }^{5}$

The close relationship between T2DM and both cardiovascular disease $(C V D)^{6}$ and chronic kidney disease (CKD) is well established. ${ }^{7,8}$ Given that hyperglycaemia promotes CVD and CKD, there used to be a presumption that tight glucose control would slow the progression of these complications. This theory, however, was challenged by the UK Prospective Diabetes Study in 1998, which suggested that glucose control did not have a major impact on large vessel disease. ${ }^{9}$ This led to a degree of nihilism among clinicians regarding glucose management, and a greater focus on managing hypertension and lipids.

The therapeutic landscape began to change in the mid-2010s with the emergence of the sodium-glucose co-transporter 2 inhibitors (SGLT2is) and glucagon-like peptide 1 receptor agonists (GLP1RAs). Large cardiovascular outcomes trials (CVOTs) demonstrated that the SGLT2is canagliflozin and empagliflozin could significantly reduce the risk of major cardiovascular events in patients with T2DM. 10,11 Furthermore, exploratory analyses suggested that all licensed SGLT2is offer heart failure benefits and protect renal function (Table 1)..$^{10-13}$

Subsequent dedicated renal and heart failure outcome trials confirmed that canagliflozin and dapagliflozin can significantly reduce the risk of renal events, 14,15 and that empagliflozin and dapagliflozin can significantly reduce the risk of hospitalisation for heart failure (Table 2). ${ }^{16,17}$

GLP1RAs have also demonstrated cardiovascular (CV) benefits in patients with T2DM. Indeed, four of the seven members of this 
Table 1 Trials demonstrating major adverse cardiovascular events or hospitalisation for heart failure benefit

\begin{tabular}{|c|c|c|c|c|}
\hline Trial* & Glucose-lowering agent & $\mathrm{CV}$ risk status of trial population & MACE HR $(95 \% \mathrm{Cl})$ & HHF HR (95\% Cl) \\
\hline \multicolumn{5}{|l|}{ SGLT2is } \\
\hline CANVAS Program ${ }^{10}$ & Canagliflozin & $66 \%$ with CVD & $0.86(0.75$ to 0.97$)$ & 0.67 (0.52 to 0.87$)$ \\
\hline VERTIS CV ${ }^{12}$ & Ertugliflozin & $100 \%$ with ACVD & $0.97(0.85$ to 1.11$) \dagger$ & 0.70 (0.54 to 0.90$)$ \\
\hline DECLARE-TIMI $58^{13}$ & Dapagliflozin & $41 \%$ with CVD & $0.93(0.84$ to 1.03$)$ & 0.73 (0.61 to 0.88$)$ \\
\hline REWIND $^{18}$ & Dulaglutide & $31 \%$ with CVD & 0.88 (0.79 to 0.99$)$ & $0.93(0.77$ to 1.12$)$ \\
\hline Harmony Outcomes ${ }^{19}$ & Albiglutide & $100 \%$ with CVD & 0.78 (0.68 to 0.90$)$ & NA \\
\hline SUSTAIN-620 & Semaglutide & $83 \%$ with CVD/CKD & 0.74 (0.58 to 0.95$)$ & $1.11(0.77$ to 1.61$)$ \\
\hline LEADER $^{21}$ & Liraglutide & High CV risk & 0.87 (0.78 to 0.97$)$ & 0.87 (0.73 to 1.05$)$ \\
\hline \multicolumn{5}{|c|}{ *Benefit in MACE or HHF as defined by a HR for which the upper $\mathrm{Cl}$ did not pass $1.00 ;+95.6 \%$ confidence interval. } \\
\hline
\end{tabular}

Table 2 Findings of dedicated renal or heart failure outcomes trials involving SGLT2is

\begin{tabular}{|c|c|c|c|c|}
\hline Trial & Glucose-lowering agent & Key baseline characteristics & Primary endpoint & $\mathrm{HR}(95 \% \mathrm{Cl})$ \\
\hline \multicolumn{5}{|c|}{ Dedicated renal outcomes trials } \\
\hline DAPA-CKD ${ }^{14 *}$ & Dapagliflozin & $\begin{array}{l}\text { Mean eGFR } \\
43.1 \mathrm{~mL} / \mathrm{min} / 1.73 \mathrm{~m}^{2}\end{array}$ & $\begin{array}{l}\text { Composite of: sustained } \\
50 \% \text { decrease in eGFR, } \\
\text { ESRD, or CV or renal death }\end{array}$ & $\begin{array}{l}0.61 \\
(0.51 \text { to } 0.72) \\
p<0.001\end{array}$ \\
\hline CREDENCE ${ }^{15}$ & Canagliflozin & $\begin{array}{l}\text { Mean eGFR } \\
56.2 \mathrm{~mL} / \mathrm{min} / 1.73 \mathrm{~m}^{2}\end{array}$ & $\begin{array}{l}\text { Composite of: doubling of } \\
\text { serum creatinine, ESRD, or } \\
\text { CV or renal death }\end{array}$ & $\begin{array}{l}0.70 \\
(0.59 \text { to } 0.82) \\
p=0.00001\end{array}$ \\
\hline \multicolumn{5}{|c|}{ Dedicated heart failure outcomes trials } \\
\hline DAPA-HF 16 * & Dapagliflozin & $\begin{array}{l}\text { Mean LVEF } \\
31.1 \%\end{array}$ & $\begin{array}{l}\text { Composite of: worsening heart } \\
\text { failure or CV death }\end{array}$ & $\begin{array}{l}0.74 \\
(0.65 \text { to } 0.85) \\
p<0.001\end{array}$ \\
\hline EMPEROR Reduced ${ }^{17}$ * & Empagliflozin & $\begin{array}{l}\text { Mean LVEF } \\
27.4 \%\end{array}$ & $\begin{array}{l}\text { Composite of: hospitalisation } \\
\text { for heart failure or } \mathrm{CV} \text { death }\end{array}$ & $\begin{array}{l}0.75 \\
(0.65 \text { to } 0.86) \\
p<0.001\end{array}$ \\
\hline \multicolumn{5}{|c|}{$\begin{array}{l}\text { Cl, confidence interval; CREDENCE, Evaluation of the Effects of Canagliflozin on Renal and Cardiovascular Outcomes in Participants with Diabetic Nephropathy } \\
\text { CV, cardiovascular; DAPA-CKD, A study to evaluate the effect of dapagliflozin on renal outcomes and Cardiovascular mortality in patients with Chronic Kidney } \\
\text { Disease; DAPA-HF, Dapagliflozin and Prevention of Adverse Outcomes in Heart Failure; eGFR, estimated glomerular filtration rate; EMPEROR, Empagliflozin } \\
\text { Outcome Trial in Patients with Chronic Heart Failure and a Reduced Ejection Fraction; ESRD, end-stage renal disease; HR, hazard ratio; LVEF, left ventricular } \\
\text { ejection fraction. }\end{array}$} \\
\hline
\end{tabular}

drug class significantly reduced the risk of major CV events in their respective CVOTs (Table 1). ${ }^{18-21}$

\section{Rationale for integrated care in type 2 diabetes mellitus} By the late 2000s, T2DM was being recognised as a vascular disease but there was no meaningful collaboration between diabetologists and specialists who were managing vascular complications. Cardi- ologists would treat people with T2DM following an acute CV event, then pass them back to primary care or a diabetologist for continued glucose management. In a similar manner, renal services would await the point at which patients with T2DM were approaching the need for dialysis before becoming involved in their care.

The needs of many people with diabetes fall between primary and secondary care, and if prevalence projections are accurate, ${ }^{1}$ sec- 
ondary care centres may not be able to cope with the sheer number of people moving through the system in the future. Expert care teams in the community may bridge the gap between the two tiers of care and relieve the pressure on secondary care systems.

The availability of medications that offer cardio-renal protection for people with diabetes has added an extra layer of complexity to managing T2DM in primary care. Close ties between primary care and local expert multidisciplinary teams (MDTs) may help to upskill primary care physicians and maximise the benefit of SGLT2is and GLP1RAs.

Local integrated MDT care has the potential to benefit people with diabetes in terms of both appointment burden and long-term health outcomes. In addition, the reduced pressure on secondary care centres and the cost savings associated with preventing CV and renal events are benefits attractive to healthcare providers.

The urgent need for integrated care for people with diabetes has been intensified by the COVID-19 pandemic. Data from English hospitals demonstrated that a third of COVID-19-related deaths occurred in people with diabetes. Furthermore, CV or renal complications were independent risk factors for COVID-19-related death in this cohort. ${ }^{22}$ In the COVID-19 era, it has become even more important that all modifiable cardio-renal risk factors are minimised in patients with diabetes to limit the risk of hospitalisation.

\section{Barriers to integrated care for people with diabetes}

Despite the growing evidence base supporting a multidisciplinary approach to diabetes management, this is not commonplace in the UK. Setting up integrated care systems within the UK National Health Service (NHS) framework can be challenging.

Sharing patient information among practitioners in different settings is a critical aspect of any putative integrated care system but difficulties with IT connectivity within the NHS represent a considerable hurdle to establishing effective MDTs.

Integrated care systems also require close ties between primary and secondary care and the commitment of the participating healthcare professionals (HCPs). Unfortunately, such close ties are not universal, the NHS internal market system can hinder the development of links between tiers of care and not all HCPs are convinced of the value and efficiency of multidisciplinary care. Integrated care systems also require secure long-term funding and a pool of HCPs with the requisite expertise and experience.

Despite these challenges, such systems have been set up successfully around the UK. Here we describe two case studies from very different healthcare environments.

\section{Key steps for setting up an integrated care system}

Although the reasons for setting up the integrated care systems described below were different, the following steps taken to overcome the barriers had much in common:

- review of the current systems to identify the key barriers to care and/or the specific unmet needs in the population;

- engagement with suitable funding bodies;

- where needed, improvement of links between primary and secondary care to ensure that all care providers are engaged and to facilitate navigation of the internal market system;
- identification and recruitment of MDT members with the requisite knowledge, skills and experience;

- acquisition and adoption to an integrated IT system that allows all care providers access to medical records and unhindered communication between the tiers of care;

- setting up of local clinics to bring the expert care closer to the community;

- implementation of mechanisms for early risk stratification and electronic triage of people who would benefit most MDT clinics.

\section{Integrated care case study 1: the South London experience \\ Geography and patient population}

Lambeth, South London, is one of the most densely populated boroughs in England and Wales. ${ }^{23}$ The population is characterised by significant ethnic diversity, young age and difficult socioeconomic circumstances. ${ }^{24}$

A typical primary care practice in London will have 500-1000 patients with T2DM, of whom between 200 and 400 may have CV risk factors and/or early evidence of CKD. ${ }^{25}$ On average, people in Lambeth develop T2DM at a younger age than those in other parts of the UK, and are predisposed to developing cardio-renal complications. ${ }^{25}$ The primary aim of the service was to address the high burden of cardio-renal risk by facilitating the early identification of high-risk patients and rapid referral to secondary care.

\section{Team structure}

The team comprises a specialist diabetes nurse, a dietician, a clinical consultant pharmacist, a primary care physician (PCP) and a diabetologist. A dedicated administrator was also recruited to manage the logistical aspects of the integrated care system.

\section{Systems and processes: pre-COVID-19}

Local pathways were established to promote the early identification and referral of patients at high risk of CKD. ${ }^{5}$ In brief, people with a confirmed estimated glomerular filtration rate (eGFR) below $30 \mathrm{~mL} / \mathrm{min} / 1.73 \mathrm{~m}^{2}$ or non-diabetic kidney disease are referred to secondary care immediately; patients with an eGFR higher than $30 \mathrm{~mL} / \mathrm{min} / 1.73 \mathrm{~m}^{2}$ are referred to the MDT if they have an urine albumin:creatinine ratio above $30 \mathrm{mg} / \mathrm{mmol}$ despite adequate renin-angiotensin-aldosterone system blockade, or if an annual fall in eGFR of more than $5 \mathrm{~mL} / \mathrm{min} / 1.73 \mathrm{~m}^{2}$ is observed. Patients are referred to the MDT for guidance on optimising treatment in the context of CKD or CVD. Patients with acute or advanced CKD and/or CVD and those who require more detailed investigation are referred to the relevant clinical service at Guy's and St Thomas' Hospital.

Multidisciplinary clinics are held weekly in four locations across Lambeth. During visits, serum creatinine, glycated haemoglobin $\left(\mathrm{Hb}_{1 \mathrm{c}}\right)$, plasma glucose and eGFR are measured the point of care to enable prompt decision-making and triage. Joint decisionmaking is a key focus of the MDT. Shared plans are developed with patients that cover lifestyle, diet, self-care and management of medications, with an emphasis on sick day rules. 
The MDT clinics use the EMIS electronic patient record platform with adapted functionality, which gives clinicians access to primary and hospital medical records including primary care prescriptions and laboratory results from both primary and secondary care. This system facilitates sharing of key information and reduces duplication of care processes. The IT platform also enables efficient virtual clinics, during which records from patients referred from local general practitioners are reviewed by the MDT, and advice and management plans are sent back to the referring clinicians.

Following an initial pilot period, the integrated care service was funded by the local clinical commissioning group, which appreciated the importance of early identification of high-risk individuals, prompt triaging and the focus on delivery of an integrated hospital- and community-based service (Figure 1).

\section{Post-COVID-19 adaptations}

At the beginning of the pandemic, the MDT processes were restructured to meet social distancing requirements, meetings were held virtually and face-to face appointments with patients were kept to a minimum. However, patients with acute clinical needs, such as rapid decline in renal function or inadequate glycaemic control that necessitated immediate administration of insulin, were prioritised for face-to-face appointments. In addition, patients with mental health problems or communication difficulties were also seen in person.

A reduced-contact service was established to run alongside the face-to-face clinics. Patients are invited to a healthcare assistant-led screening clinic during which $\mathrm{HbA}_{1 \mathrm{c}}$, blood pressure, cholesterol, serum creatinine, urine albumin, body mass index, foot health and smoking habits are measured and recorded electronically at the point of care. In addition, patients are able to add their own blood glucose readings to their records using the DIASEND platform. The complete electronic records and results are reviewed by the MDT, and patients were contacted by phone a week later to discuss results and treatment plans.

Community renal diabetes clinics are held weekly. Eight face-toface appointments and 18 telephone/virtual consultations are available, the latter being appropriate for patients for whom the key information described above is already available. In addition, 12 injectable medication starter sessions are provided weekly by specialist nurses.

\section{Integrated care case study 2: the Western Health and Social Care Trust experience}

\section{Geography and patient population}

The Western Trust serves a population of approximately 300,000 people in Northern Ireland and covers an area of $4842 \mathrm{~km}^{2}$, which encompasses the counties of Derry/Londonderry, Tyrone and Fermanagh. In contrast to the population of Lambeth, more than half of the people served by the Western Trust reside outside the three main population centres. There are 49 general practices within the Western Trust that care for approximately 14,500 patients with diabetes, and acute complications of diabetes are managed at two hospitals that lie 45 miles apart.

An acute shortage of consultants in 2012 led to a reassessment of diabetes services in the Western Trust. The essential service review revealed long waiting lists for, and waiting times at, consultant-led clinics, failings in shared-care arrangements with primary care and duplication of services, all highlighting the need for a service redesign.

Figure 1. Patient flow through the Lambeth integrated diabetes care pathway for renal disease

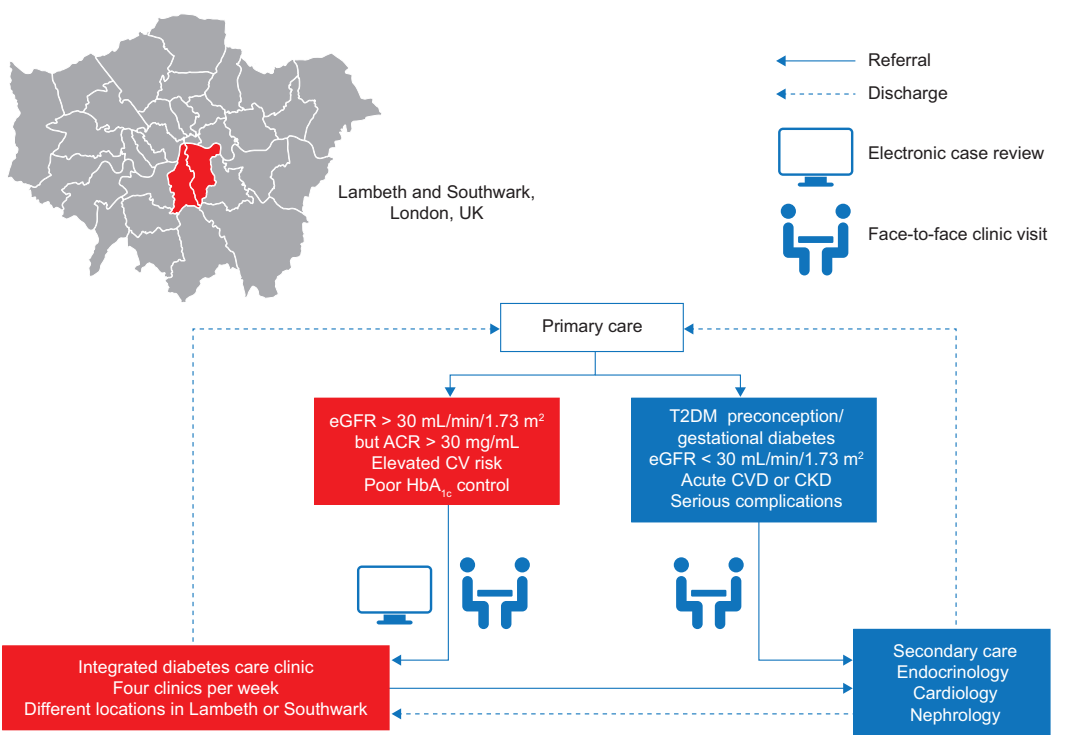

\begin{tabular}{|l|}
\hline Key innovations \\
\hline $\begin{array}{l}\text { Analysis of population care } \\
\text { needs and clinical priorities }\end{array}$ \\
\hline $\begin{array}{l}\text { Assembly of MDT to sit between } \\
\text { primary and secondary care }\end{array}$ \\
\hline Local MDT cardio-renal clinics \\
\hline $\begin{array}{l}\text { POC measurement of HbA1c } \\
\text { and serum creatinine }\end{array}$ \\
\hline $\begin{array}{l}\text { Electronic referral, case review } \\
\text { and triage }\end{array}$ \\
\hline
\end{tabular}

$A C R$, albumin:creatinine ratio; CKD, chronic kidney disease; CV, cardiovascular; CVD, cardiovascular disease; eGFR, estimated glomerular filtration rate; $\mathrm{HbA}_{1}$ c, glycated haemoglobin; MDT, multidisciplinary team; POC, point of care; T2DM, type 2 diabetes mellitus. 


\section{Team structure}

To relieve the pressure on secondary care, a community-based diabetes specialist team (DST) was set up, comprising diabetologists, nurses, dieticians, podiatrists, podiatry assistants, pharmacists, psychologists, exercise professionals and clerical staff. Funding from 'Transforming Your Care for Integrated Care Partnerships' allowed additional staff to be recruited to the DST.

\section{Systems and processes}

Several types of clinic are now run across the Western Trust to meet the different needs of patients with diabetes. On a weekly basis: nine consultant-led clinics are held at the three main hospital sites; 15 joint clinics involving diabetes specialist nurses and dieticians are held at local primary care centres and the three hospital sites; four clinics specifically for patients newly diagnosed with diabetes are held at local centres; and an additional 10 clinics led by diabetes specialist nurses are held in local primary care centres.

The Western Trust was an early adopter of the Northern Ireland Electronic Care Record system. This allowed the creation of a Trustwide central electronic portal for triaged referral from primary care to any of the DST clinics described above and the standardisation of treatment strategies and referral pathways across the Trust. Primary care physicians can upload information including notification of a new diagnosis, $\mathrm{HbA}_{1 \mathrm{c}}$, eGFR and presence of $\mathrm{CV}$ risk factors or complex complications and can request advice on the most appropriate treatments. This information can be reviewed to ensure that patients are referred to the most suitable clinic and that medication advice can be sent directly to the referring primary care physician.

Setting up the DST was not without obstacles. Joint clinic codes and reporting methods proved difficult to align, owing to an ageing patient administration system; recruitment difficulties due to temporary funding arrangements with integrated care providers and overcoming resistance to change among some staff members were, and remain, challenging issues.

Patients have benefited in several ways: offering joint clinics in a planned pathway reduced the number of appointments; the electronic triage system allowed Trust-wide referrals to be processed more quickly and updated treatment regimens to be planned and circulated more efficiently; and sharing treatment plans and discussions of next treatment steps has had a beneficial educational bonus. In addition, risk stratification has improved waiting times with new referral and recall dates now on target in some areas and reduced to a delay of 2-6 months in others (Figure 2).

\section{Discussion}

New glucose-lowering drug classes with proven CV and renal benefits are shifting the paradigm of diabetes care from management of glycaemia to protection of organ function. At the same time, the rising prevalence of T2DM is placing considerable pressures on healthcare systems. This combination of factors makes the care of people with T2DM in the primary care setting more complex than ever before. The often-siloed system of primary care and secondary care is not suited to the management of this rapidly growing population whose needs span the two care settings.

Despite the obstacles, the two case studies above show how innovative integrated systems can bring multidisciplinary diabetes care to the community, resulting in shorter waiting times, less duplication of procedures, less pressure on secondary care, enhanced opportunities to educate both people who are treated and HCPs and, in all likelihood, better disease outcomes.

The different approaches taken by the teams in Lambeth and

Figure 2. Patient flow through the Western Trust integrated diabetes care programme

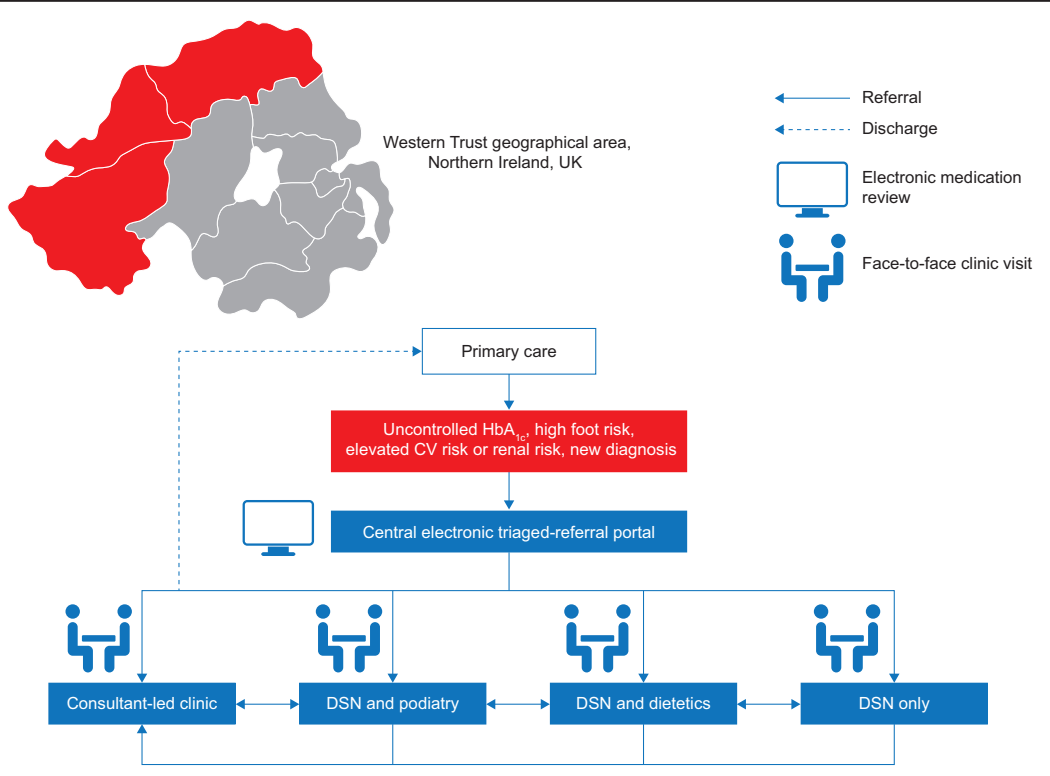

\begin{tabular}{|l|}
\hline Key innovations \\
\hline $\begin{array}{l}\text { Analysis of population care } \\
\text { needs }\end{array}$ \\
\hline $\begin{array}{l}\text { Assembly of MDT to sit between } \\
\text { primary and secondary care }\end{array}$ \\
\hline $\begin{array}{l}\text { Several specialist clinics to meet } \\
\text { needs }\end{array}$ \\
\hline $\begin{array}{l}\text { Electronic referral, case review } \\
\text { and triage }\end{array}$ \\
\hline
\end{tabular}

CV, cardiovascular; DSN, diabetes specialist nurse; $\mathrm{HbA}_{1}$, glycated haemoglobin; MDT, multidisciplinary team. 


\section{Key messages}

- The increasing prevalence of diabetes in the UK is likely to lead to a corresponding increase in the prevalence of diabetes-related cardio-renal disease

- The traditional two-tier care model may not be suitable for, nor capable of meeting, the complex needs of people with diabetes

- In response to long waiting times in secondary care and because of a need to detect and intervene at an earlier stage in people at high risk of kidney disease, respectively, healthcare professionals in Northern Ireland and South London have established alternative care models

- Key features common to both models include building dedicated multidisciplinary teams, regular local clinics in the community, early identification of cardio-renal risk, shared electronic medical records and virtual clinics

the Western Trust reflect the different needs of the populations they serve and suggest that a 'one size fits all' approach is not appropriate. However, there are several factors that appear to be pivotal to the success of such systems: IT platforms that allow information to be shared among HCPs; flexible referral processes that allow easy movement of patients to the most appropriate clinic; and, perhaps most importantly, the availability of dedicated specially trained HCPs.

The COVID-19 pandemic has provided a stark reminder of the wider health implications of diabetes, and highlighted the importance of protecting CV and renal function in those with this condition. Integrated expert care can not only facilitate early identification of people at risk but also maximise the benefits of new treatments that can reduce the risk of developing devastating $\mathrm{CV}$ or renal complications.

The flexibility built into the integrated care system in Lambeth enabled it to adapt quickly to the changing circumstances brought about by COVID-19. Community-based expert care prevented patients needing to travel to under-pressure higher-risk secondary care centres and allowed the management of complex diabetes to continue in a risk-managed setting.

The combination of increasing numbers of patients with diabetes and a rapidly evolving treatment landscape has placed unprecedented pressure on healthcare systems and exposed the limitations of established care models. However, innovative integrated community-based care programmes can relieve the pressure on healthcare systems, maximise the benefits offered by new treatments and improve outcomes for people with diabetes.

Authors' contributions: All authors have contributed to the writing or reviewing of this manuscript.

Acknowledgements: Medical writing support was provided by Dr Matthew Long of PharmaGenesis, Cardiff, UK
Conflict of interest: SB: has been a senior clinical academic since 1993 and since that time reports having received honoraria, teaching and research sponsorship/grants from the following: Abbott, AstraZeneca, Boehringer Ingelheim, Bristol Myers Squibb, Cellnovo, Diartis, Eli Lilly, GlaxoSmithKline, Merck Sharp and Dohme, Novartis, Novo Nordisk, Pfizer, Roche, Sanofi-Aventis, Schering-Plough, Servier and Takeda; received funding for the development of educational programmes from Cardiff University, Doctors.net, Elsevier, Onmedica, Omnia-Med and Medscape; owns a share of Glycosmedia which carries sponsorship declared on site. JK: attended scientific advisory boards for and received honoraria or consulting fees from Eli Lilly, Novo Nordisk, Sanofi, Napp, AstraZeneca and Boehringer Ingelheim; received grants/research support from Sanofi and AstraZeneca. EC: None. NB: received speaker honoraria from AstraZeneca, Boehringer Ingelheim, Merck Sharp and Dohme, Novo Nordisk and Sanofi, and educational support for conference attendance from Novo Nordisk and Sanofi. LH: None. MC: None. AH: None. AR: None.

Funding: Medical writing support was funded by Napp Pharmaceuticals.

\section{References}

1. International Diabetes Federation. IDF diabetes atlas 2017 [cited 2019 November]. Available from: https://www.diabetesatlas.org/en/resources/

2. Evans JM, Newton RW, Ruta DA, et al. Socio-economic status, obesity and prevalence of type 1 and type 2 diabetes mellitus. Diabet Med 2000;17(6):478-80.

3. Bruno G, Runzo C, Cavallo-Perin P, et al. Incidence of type 1 and type 2 diabetes in adults aged 30-49 years: the population-based registry in the province of Turin, Italy. Diabetes Care 2005;28(11):2613-19. https://doi.org/10.2337/diacare.28.11.2613

4. Holman N, Young B, Gadsby R. Current prevalence of type 1 and type 2 diabetes in adults and children in the UK. Diabet Med 2015;32(9):111920. https://doi.org/10.1111/dme.12791

5. Saito I, Kokubo Y, Yamagishi K, et al. Diabetes and the risk of coronary heart disease in the general Japanese population: The Japan Public Health Center-based prospective (JPHC) study. Atherosclerosis 2011; 216(1):187-91. https://doi.org/10.1016/j.atherosclerosis.2011.01.021

6. International Diabetes Federation. Diabetes and cardiovascular disease 2016 [cited 2019 January]. Available from: www.idf.org/cvd.

7. Dean J. Organising care for people with diabetes and renal disease. J Ren Care 2012;38(Suppl 1):23-9. https://doi.org/10.1111/j.1755-6686.2012. 00272.x

8. King P, Peacock I, Donnelly R. The UK Prospective Diabetes Study (UKPDS): clinical and therapeutic implications for type 2 diabetes. $\mathrm{Br} \mathrm{J}$ Clin Pharmacol 1999;48(5):643-8. https://doi.org/10.1046/j.1365-2125. 1999.00092.x

9. UK Prospective Diabetes Study Group. Intensive blood-glucose control with sulphonylureas or insulin compared with conventional treatment and risk of complications in patients with type 2 diabetes (UKPDS 33). Lancet 1998;352(9131):837-53.

10. Neal B, Perkovic V, Mahaffey KW, et al. Canagliflozin and cardiovascular and renal events in type 2 diabetes. N Engl J Med 2017;377(7):644-57. https://doi.org/10.1056/NEJMoa1611925

11. Zinman B, Wanner C, Lachin JM, et al. Empagliflozin, cardiovascular outcomes, and mortality in type 2 diabetes. N Engl J Med 2015; 373(22):2117-28. https://doi.org/10.1056/NEJMoa1504720

12. Cannon CP, Pratley R, Dagogo-Jack S, et al. Cardiovascular outcomes with ertugliflozin in type 2 diabetes. N Engl J Med 2020;383(15):142535. https://doi.org/10.1056/NEJMoa2004967

13. Wiviott SD, Raz I, Bonaca MP, et al. Dapagliflozin and cardiovascular outcomes in type 2 diabetes. N Engl J Med 2019;380(4):347-57. https://doi.org/10.1056/NEJMoa1812389

14. Heerspink HJL, Stefansson BV, Correa-Rotter R, et al. Dapagliflozin in patients with chronic kidney disease. N Engl J Med 2020;383(15):1436-46. https://doi.org/10.1056/NEJMoa2024816 
15. Perkovic V, Jardine MJ, Neal B, et al. Canagliflozin and renal outcomes in type 2 diabetes and nephropathy. N Engl J Med 2019;380(24):2295-306. https://doi.org/10.1056/NEJMoa1811744

16. McMurray JJV, Solomon SD, Inzucchi SE, et al. Dapagliflozin in patients with heart failure and reduced ejection fraction. N Engl J Med 2019; 381:1995-2008. https://doi.org/10.1056/NEJMoa1911303

17. Packer M, Anker SD, Butler J, et al. Cardiovascular and renal outcomes with empagliflozin in heart failure. N Engl J Med 2020;383(15):141324. https://doi.org/10.1056/NEJMoa2022190

18. Gerstein HC, Colhoun HM, Dagenais GR, et al. Dulaglutide and cardiovascular outcomes in type 2 diabetes (REWIND): a double-blind, randomised placebo-controlled trial. Lancet 2019;394(10193):121-30. https://doi.org/10.1016/S0140-6736(19)31149-3

19. Hernandez AF, Green JB, Janmohamed $S$, et al. Albiglutide and cardiovascular outcomes in patients with type 2 diabetes and cardiovascular disease (Harmony Outcomes): a double-blind, randomised placebo-controlled trial. Lancet 2018;392(10157):1519-29. https://doi.org/10.1016/ S0140-6736(18)32261-X

20. Marso SP, Bain SC, Consoli A, et al. Semaglutide and cardiovascular out- comes in patients with type 2 diabetes. N Engl J Med 2016; 375(19):1834-44. https://doi.org/10.1056/NEJMoa1607141

21. Marso SP, Daniels GH, Brown-Frandsen $K$, et al. Liraglutide and cardiovascular outcomes in type 2 diabetes. N Engl J Med 2016;375(4):31122. https://doi.org/10.1056/NEJMoa1603827

22. Holman N, Knighton P, Kar P, et al. Risk factors for COVID-19-related mortality in people with type 1 and type 2 diabetes in England: a population-based cohort study. Lancet Diabetes Endocrinol 2020;8(10):82333. https://doi.org/10.1016/S2213-8587(20)30271-0

23. Office for National Statistics. Population estimates for the UK 2019 [cited 2019 December]. Available from: https://www.ons.gov.uk/peoplepopulationandcommunity/populationandmigration/populationestimates/datas ets/populationestimatesforukenglandandwalesscotlandandnorthernireland.

24. Lambeth Council. Demography fact sheet 2017 [cited 2019 November]. Available from: https://www.lambeth.gov.uk/sites/default/files/ssh-demography-factsheet-2017.pdf.

25. Chamley M. Community diabetes in South London. $\mathrm{Br} J$ Diabetes 2015;15(2):78-82. http://dx.doi.org/10.15277/bjdvd.2015.013

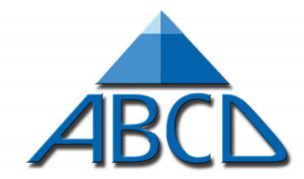

Association of British Clinical Diabetologists

\section{ABCD DIY APS Audit NOW LIVE!}

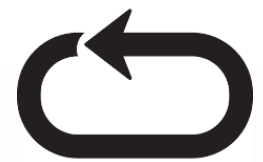

The ABCD Do-It-Yourself Artificial Pancreas System (DIY APS) audit is now live

These user developed systems are unregulated and unapproved, but users report improved time-in-range, improved $\mathrm{HbA}_{1 \mathrm{c}}$ and massive improvements in quality of life.

\section{We NEED data from all centres with ANY users of these systems to help provide robust evidence and guide future practice.}

\section{Ask yourself "Do we have a DIY looper in our service?"}

\section{If YES please sign up here ... https://abcd.care/DIYAPS}

Participation in this audit is currently best practice as recommend by the Diabetes UK position statement Our online tool is secure, GDPR compliant, encrypted and anonymised - patient details are only viewable by your centre 